\title{
A COMPLETE STOKES VECTOR POLARIMETER
}

\author{
FRANK Q. ORRALL \\ Institute for Astronomy, University of Hawaii, Honolulu, Hawaii 96822, U.S.A.
}

\begin{abstract}
A scanning photoelectric polarimeter of $16 \mathrm{~cm}$ aperture that can measure all four Stokes parameters of the visible radiation of the Sun's disk, the Corona, Moon and planets, has been constructed at the Institute for Astronomy and is installed on Mt. Haleakala. It is a two (orthogonal) channel system and uses a rotating $\lambda / 4$ plate modulator. Photon counting is done by a digital computer that also Fourier analyzes the modulated output of the photomultipliers, and, from the Fourier components, computes the Stokes parameters in real time.
\end{abstract}

\section{Introduction}

Most of our knowlèdge of the physical nature of astronomical objects has been inferred from the intensity of the radiation $I(\lambda)$ received from them. But of course a complete description of the radiation (complete except for absolute phase) requires a measurement of the complete Stokes' vector $\{1, Q, U, V\}$. The parameters $Q(\lambda)$, $U(\lambda)$ and $V(\lambda)$ contain fundamental information about the magnetic fields, non-thermal processes, anisotropies in the source and the process of radiative transfer that can be found in no other way. Although a number of polarization measurements have been made on the Sun, few have measured all four Stokes' parameters except for some special purpose as in the vector magnetograph. At the Institute for Astronomy we have recently designed and constructed a complete Stokes' vector polarimeter-photometer that can be used to scan the Sun's disk and corona as well as the Moon and planets. At present, it uses broad or narrow band filters to isolate regions of the spectrum. Later, a high resolution scanning monochrometer will be added so that the complete Stokes' four-vector can be measured as a function of wavelength.

\section{The Instrument}

A schematic drawing of the optics and electronics is shown in Figure 1. The telescope is a coronagraph of $16 \mathrm{~cm}$ aperture and $250 \mathrm{~cm}$ focal length $(f / 15)$. Two interchangeable telescope objectives are provided; one a singlet lens of coronagraph quality, the other an achromat. A $2.5 \mathrm{~cm}$ image of the Sun is formed on a conical surface with a small scanning aperture at its apex, and this conical surface reflects all of the light that does not pass through the aperture into baffles along the side of the telescope tube. It thus serves in place of an occulting disk. A second objective behind the aperture, collimates the light into a beam $2 \mathrm{~cm}$ in diameter. Immediately behind this is a modulator consisting of a rotating $\lambda / 4$ plate and a polarizing beamsplitter (Wollaston prism). The two collimated beams from the beamsplitter fall on separate photomultipliers, which can be cooled by dry ice to reduce thermal noise. Suitable glass, gelatin or interference filters can be placed in front of the photomultiplier windows to isolate broad or narrow regions of the spectrum. 
The entire optical system fits in a cylindrical tube $22.5 \mathrm{~cm}$ in diameter and 4.5 meters long (including the dust tube in front of the objective). This tube is mounted on the 12-ft solar spar on Mt. Haleakala, Maui, at an elevation of $3054 \mathrm{~m}$. The spar is pointed at the center of the Sun, but the entire polarimeter moves relative to the spar to scan the Sun or corona. Thus the polarimeter is always used on-axis. An aperture stop and mask are suitably placed in front of the collimator to make the instrument in all respects a Lyot coronagraph, provided that the singlet, low-scatter objective is used. If the scanning aperture used in the primary focal plane is small, a field lens (usually required in the Lyot coronagraph) is not needed.

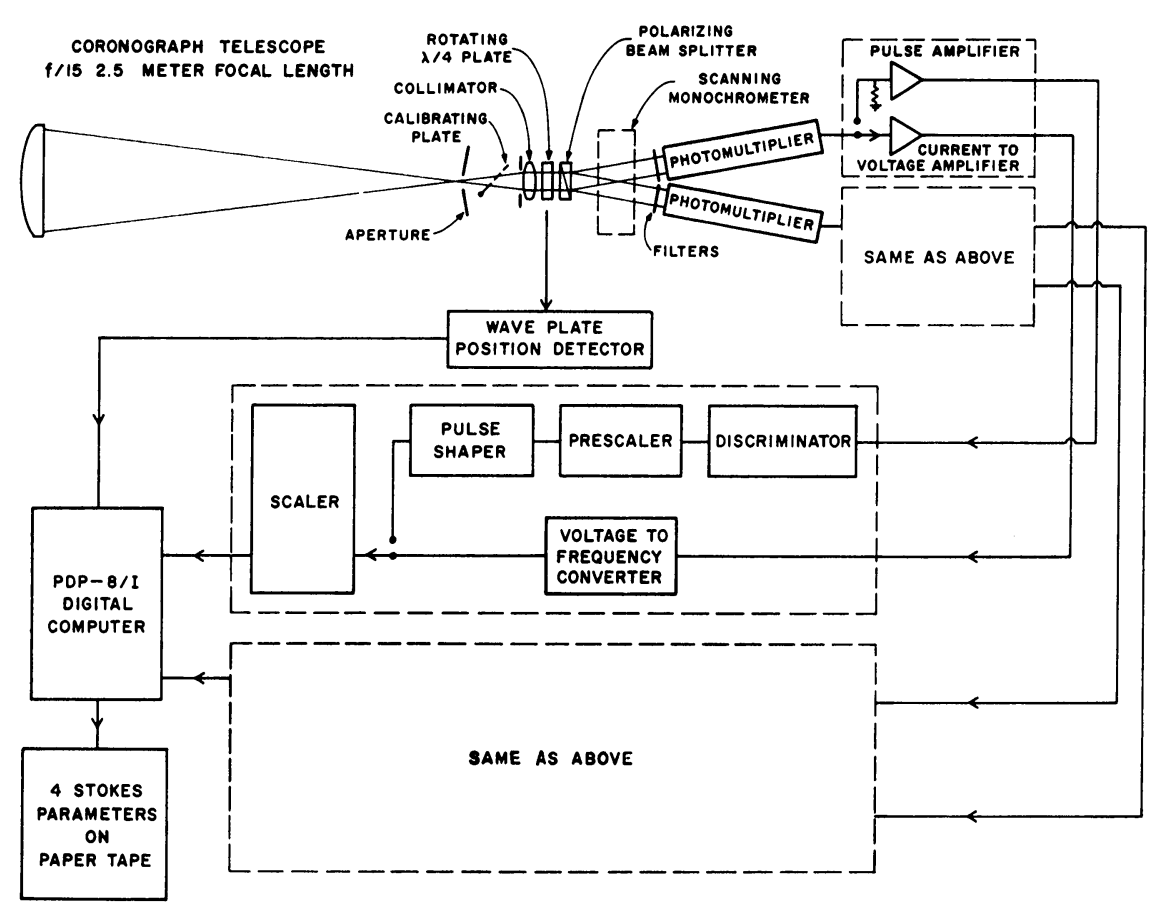

Fig. 1. A schematic diagram of the optics and electronics of the complete Stokes vector polarimeter of the Institute for Astronomy.

A PDP-8/I digital computer points and controls the instrument, receives and processes data from it, and prints out the processed data on a typewriter or on high speed punched paper tape. The polarimeter will share time on the computer with other observatory instruments.

\section{A. Scanning in the Plane of The SKy}

The 12-ft Solar Spar on which the polarimeter is mounted remains photoelectrically pointed at the center of the Sun's disk to accommodate the other instruments mounted on it. Since the polarimeter scanning aperture is always on-axis in order to minimize 
instrumental polarization, the polarimeter must move relative to the spar in order to scan on the plane of the sky. This is accomplished by mounting the polarimeter on gimbals near its center of mass, and then driving its forward end in two coordinates by means of two precision screws driven by stepping motors. These stepping motors are under direct computer control, so that a raster scan, or any other type of scan can be performed on the plane of the sky by suitably programming the computer.

\section{B. THE MODULATOR}

The modulator is driven by a stepping motor geared so that 160 steps of the motor produces one full rotation of the $\lambda / 4$ plate. The pulses for the stepping motor are provided (through a translator) by the kilocycle time base of the computer. Thus at $10^{3}$ steps/sec the $\lambda / 4$ plate rotates 6.25 times per second. Most polarimeters or magnetometers have modulators based on either the Pockels, Kerr or Piezo-optical effects and can be driven at frequencies from $10^{3}$ to $10^{6} \mathrm{~Hz}$. The low modulation frequency $(6.25 \mathrm{~Hz})$ of our polarimeter has some disadvantages, but it enables us to use digital methods throughout. This makes it possible to detect and correct for spurious modulation which would otherwise be interpreted as polarization.

The computer keeps track of the angular position of the wave plate by counting steps. To insure that this count has not been lost, a small photoelectric sensor detects a fiducial mark on the edge of the wave-plate at each full rotation of the plate and the computer produces an error alarm if this does not come at the expected time.

\section{Electronics}

The output of the photomultiplier can be recorded in two alternate ways: the pulses produced by individual photons can be counted; or the photocurrent produced by a beam of photons can be measured. The pulsewidth is such that if more than about $5 \times 10^{6}$ pulses arrive each second, enough coincidences occur that the counting rate is no longer proportional to the photon arrival rate. Thus if the counting frequency exceeds about 5 megacycles, it becomes necessary to measure photocurrent instead of counting photons. Since this instrument is intended to be used over a wide range in intensities, both types of measuring systems are required.

As shown in Figure 1, each of the two photomultipliers has a separate channel of electronics, and each of these consists of two parallel (alternate) systems. Each pulse counting system has a pulse amplifier, and a discriminator which must be set at an appropriate level to distinguish between noise and true pulses due to photons. The current measuring systems consist of a current to voltage amplifier followed by a voltage to frequency converter. Thus each system produces counting rates that are proportional to the intensity of the light beam falling on the photocathode. In both systems the actual counting is done in the PDP-8/I computer.

\section{Theory}

Let $\mathbf{S} \equiv\{I, Q, U, V\}$ be the Stokes vector of the incident beam of light to be measured, 
and let $\mathbf{S}^{\prime}=\left\{I^{\prime}, Q^{\prime}, U^{\prime}, V^{\prime}\right\}$ represent the beam as modified by passing through the linear retarder (wave plate) and the linear polarizer (Wollaston prism). The transformation from $\mathbf{S}$ to $\mathbf{S}^{\prime}$ can be expressed by the Mueller calculus:

$$
\mathbf{S}^{\prime}=\left[M_{P}\right]\left[M_{R}\right] \mathbf{S}
$$

Here $\left[M_{P}\right]$ and $\left[M_{R}\right]$ are the Mueller matrices of the polarizer and retarder respectively. They have a simple well known form if the retarder and polarizer are linear and ideal (Shurcliff, 1962).

The matrix $\left[M_{R}\right]$ will depend on the retardance $\delta$, and on the instantaneous angle of orientation $\psi$ of the fast axis of the wave plate. If the plate rotates at circular frequency $\omega$ then $\psi=\omega t$, and $\left[M_{R}\right]$ will be a function of time. We choose for simplicity the convention that $\psi=0$ when the normalized fast eigenvector of the wave plate is $\{1,1,0,0\}$. We require only the intensity of the light that falls on the photocathode that is, the first component or parameter of $\mathbf{S}^{\prime}$, namely $I^{\prime}$. From Equation (1) we can readily find that

$$
2 I^{\prime}=I \pm[(a+b \cos 4 \omega t) Q+(b \sin 4 \omega t) U+(c \sin 2 \omega t) V]
$$

where

$$
\begin{aligned}
& a \equiv \frac{1}{2}(1+\cos \delta) \\
& b \equiv \frac{1}{2}(1-\cos \delta) \\
& c \equiv \sin \delta .
\end{aligned}
$$

In Equation (2) and in what follows, the + sign applies to the beam from the Wollaston prism whose normalized eigenvector is $\{1,1,0,0\}$ and the - sign to the other beam with the eigenvector $\{1,-1,0,0\}$. The retardance $\delta$ of the wave plate will only nominally be $90^{\circ}$, and $a, b$ and $c$ can be considered constants of the instrument that will vary with the wavelength distribution of the light being measured. We note from Equation (2) that if $\delta$ approaches $180^{\circ}$, the parameter $V$ will be indeterminate.

After correcting the observed photocurrents for dark current and multiplying them by suitable calibration constants, their time dependent value $g(\psi=\omega t)$ should equal the right hand side of Equation (2). In practice we fit $g(t)$ to a Fourier series

$$
g(t)=A_{0}+\sum_{n=1}\left[A_{i} \cos n \omega t+B_{i} \sin n \omega t\right]
$$

with $A_{3}=B_{3}=A_{n}=B_{n}=0$ for $n \geqslant 5$. Comparing (4) with (2) we should expect (ideally):

$$
\begin{aligned}
& A_{0}=I+a Q \\
& A_{1}=B_{1}=0 \\
& A_{4}= \pm b Q \\
& B_{4}= \pm b U \\
& B_{2}= \pm c V .
\end{aligned}
$$

The Stokes' parameters $I, Q, U, V$ can be inferred from the observed values of $A_{0}$, $B_{2}, A_{4}, B_{4}$, using either photomultiplier channel. 
In practice, of course, the other Fourier coefficients are not equal to zero by amounts that cannot be explained by noise, indicating that there are spurious sources of modulation (besides instrumental polarization) which must also contribute to $A_{0}, A_{4}$, $B_{4}$ and $B_{2}$. Our experience suggests that these sources become important when one attempts to detect polarizations of less than about $0.1 \%$ and thereafter become increasingly important. We shall mention several possible mechanisms for this spurious modulation but shall postpone a discussion of their magnitude until the instrument has been more fully tested.

\subsection{THE RETARDER OR POLARIZER MAY BE NON-LINEAR}

That is, the fourth Stokes' parameter of its eigenvectors may be significantly different from zero. Thus, for example, a wave plate made of quartz will in general be an elliptical retarder unless it is made from a section cut parallel to the optic axis of the crystal. Proper construction of the optics can minimize this.

\subsection{THE RETARDER OR POLARIZER MAY BE NON-PERFECT}

An ideal linear polarizer would have only one eigenvector. In general, however, the minor eigenvector is non-zero. Further, the retarder may be dichroic, that is, its optical transmission may depend on the form of the incident polarized light. Mica wave plates have appreciable linear dichroism, and quartz shows circular dichroism. Sekera (1956) and Hodgdon (1965) have considered some effects of non-perfect components on the modulation of a rotating wave-plate polarimeter. Compound retarders can be constructed having very little dichroism (Evans, 1970).

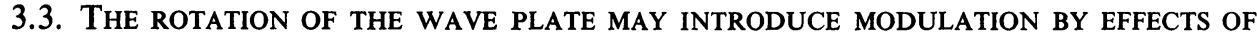 GEOMETRIC OPTICS}

If the two surfaces of the wave plate are not flat and parallel, or if the mechanical axis of rotation is not parallel to the optic axis, the collimated beam will move about on the photocathode. Since the transmission of the photomultiplier window, or the sensitivity of the photocathode, may be non-uniform, this will produce a modulation. Or, if there is a bit of dirt on wave-plate its shadow will move about on the photosurface. These effects will produce a modulation not only at the fundamental frequency $\omega$ (so that $A_{1}$ and $B_{1}$ will be non-zero) but also at higher harmonics. This modulation can be minimized by careful construction and alignment of the wave plate but is difficult to reduce it to a negligible value when measuring small amounts of polarization.

It is possible to modify Equation (2) to include all of the above effects. This will lead to a more complicated set of relations between the observed Fourier coefficients and the Stokes parameters than in Equation (5), and will include several instrumental calibration constants. These constants can be determined by calibration, and with them, the Stokes parameters can be determined in real time in the computer. We shall postpone a more complete discussion until we have better measurements of the magnitudes of these effects.

The complete vector $\mathbf{S}$ can be derived from either channel as we have done above, 
but this does not make use of the fact that the two channels contain orthogonal information. By adding and subtracting the observed values of $I^{\prime}$ from the two channels, one can derive each Stokes parameter using data obtained simultaneously. As Lyot showed, such two channel observations can reduce the effects of seeing and certain other types of noise. This can be accomplished using computer software.

\section{Sensitivity}

The accuracy or limiting polarizations that this instrument can attain will depend on the magnitudes of the photon noise, instrumental polarization, effects due to the Earth's atmosphere, spurious sources of modulation discussed above, and (for low counting rates) on the dark current. Only the photon noise can be predicted with confidence. As an example, consider the Sun's disk. Let $N_{\lambda}$ be the number of photons counted each second with an average energy $h c / \lambda$ within an effective band pass $\Delta \lambda$ by one of the photomultipliers. Then evidently

$$
N_{\lambda}=\left(f_{\lambda} A a \Delta \lambda \lambda e\right) /\left(a_{0} h c\right) \text {, }
$$

where $f_{\lambda}$ is the flux from the Sun's disk falling on the outside of the Earth's atmosphere; $A \simeq 176 \mathrm{~cm}^{2}$ is the effective area of the telescope objective; $a$ and $a_{0}=2.9 \times 10^{6}$ are the areas of the scanning aperture and of the Sun's disk respectively, given in $(\operatorname{arcs})^{2}$. The efficiency factor $e \approx 0.02$ includes the detector efficiency, the transmission of the Earth's atmosphere and optical losses at $\lambda 5000$, as well as a factor $\frac{1}{2}$ for the beam splitter. At $\lambda 5000, f_{\lambda}=200 \mathrm{erg} \mathrm{cm}^{-2} A^{-1} \mathrm{~s}^{-1}$ and

$$
N_{\lambda 5000}=6 \times 10^{7} \text { a } \Delta \lambda \text { photons s }{ }^{-1} .
$$

Thus with $\Delta \lambda=10 \AA$ and a $3 \times 3$ arc s scanning aperture, the counting rate will be $\sim 5 \times 10^{9}$ photons $\mathrm{s}^{-1}$ which will produce about the maximum photocurrent (50 $\mu \mathrm{amp}$ ) that the photomultipliers can tolerate.

In this case, if photon noise were the limiting factor, one could detect polarization of $\sim 10^{-5}$ in one second. Laboratory tests show that instrumental or modulation effects are not important if the polarization is greater than $\sim 10^{-3}$, but the lower limit set by these effects can only be determined by extended tests on the Sun's disk.

\section{Acknowledgements}

This instrument was constructed as part of a program of J. T. Jefferies, J. B. Zirker and the author to study magnetic fields and fast particles in the Sun. This work has been supported by Project Themis through ONR grant N00014-68-A0149. R. Zane designed the electronic control and data gathering systems, supervised their construction, and with J. Harwood and H. Yee wrote the necessary computer programs. J. Barclay and H. Boesgaard designed the mechanical system which was constructed under the supervision of L. Emarine and K. Miller. 


\section{References}

Evans, J. W.: 1970, (Private Communication).

Hodgdon, E. B.: 1965, Appl. Opt. 4, 1479.

Sekera, Z.: 1956, Adv. Geophys. 3, 43.

Shurcliff, W. A.: 1962, Polarized Light, Harvard University Press, Cambridge, Mass.

\section{Discussion}

Severny: (1) What is the minimal degree of polarization you could measure?

(2) Your scheme seems to be very similar to that described by Landestreet in Astrophys. J., June 1970 and used for the measurements of the magnetic field in white dwarfs.

(3) What is the difference between your scheme and the scheme proposed by Lyot and used by Dr. Dollfus?

(4) How can you discriminate polarization due to magnetic fields from that due to scattering in the process of line formation?

Orrall: To answer your questions in order:

(1) We hope that when sufficient photons can be counted the smallest measurable polarization will be between $10^{-4}$ and $10^{-5}$, a limit set by instrumental and sky polarization. But the instrument is not fully tested and we shall see.

(2) I think that his scheme uses also a rotating wave plate but I am not sure.

(3) Dr. Dollfus' instrument is also a two-channel polarimeter using a refractor on axis. It differs from ours in that it is designed to measure linear polarization and rotates about its optical axis to determine the direction of polarization. It uses a different modulation scheme from ours, and treats the output of the photomultipliers in an entirely different way.

(4) The problem of inferring the magnetic field from measured polarization is a difficult one that is not yet satisfactorily solved. We hope that this instrument will contribute to the solution of that problem. When it is we will be able to call our polarimeter a magnetometer!

Gaizauskas: It does not necessarily follow that the use of a much higher frequency of modulation of an EOLM in a photoelectric magnetograph produces a better field measurement. With modern systems, the major source of signal noise is produced by photon noise in the photomultipliers and by image motion. Photon noise can be quickly filtered out, but the power spectrum of image motion rises rapidly towards low frequencies $(10 \mathrm{~Hz}$ and less). In areas of rapidly changing field strength, the major noise component is therefore produced by image motion. If image motion is monitored at the same time as the magnetic field is rapidly scanned, it becomes possible to reject the measurements made during periods of poor seeing to improve the data.

Orrall It is certainly true that image motion will be a major source of noise when measuring regions where the field is changing rapidly. Monitoring the seeing as you suggest is certainly a good idea. But I think that a high modulation frequency is still valuable 